\title{
c
}

Revista Competitividade e Sustentabilidade - ComSuS Journal of Competitiveness and Sustainability - ComSus

e-ISSN:2359-5876

\section{ASPECTOS DO CRESCIMENTO ECONÔMICO E DO DESENVOLVIMENTO HUMANO NO MUNICÍPIO DE JAMBEIRO NA \\ DÉCADA DE 2000}

Bruna Braun Machado ${ }^{1}$

Edson Trajano Vieira ${ }^{2}$

\begin{abstract}
Resumo: Essa pesquisa baseia-se na análise do crescimento econômico e do seu impacto sobre o desenvolvimento humano no município de Jambeiro/SP durante da década de 2000 . Seu objetivo é investigar se o avanço da economia local está acompanhado da melhoria de qualidade de vida da população. O estudo está fundamentado nas teorias contemporâneas que conceituam os termos crescimento e desenvolvimento. A investigação tomou como base o perfil do crescimento econômico através da variação do PIB e PIB per capita e, em paralelo, o perfil do desenvolvimento humano por meio da variação do Índice de Desenvolvimento Humano Municipal - IDHM entre os anos de 2000 e 2010. Logo após a comparação entre os indicadores econômicos e sociais, os resultados revelam que, por um lado, a economia cresce de forma expressiva. No entanto, no que se refere à melhoria da qualidade de vida da população, observa-se melhoria nos níveis de educação, porém a conjuntura social ainda é composta por exclusão social, refletida na má distribuição de renda entre a população.
\end{abstract}

Palavras-Chave: Crescimento Econômico. Desenvolvimento Humano. Jambeiro.

\section{ASPECTS OF ECONOMIC GROWTH AND HUMAN DEVELOPMENT IN THE CITY OF JAMBEIRO DURING THE 2000S}

\begin{abstract}
This research is based on the analysis of economic growth and its impact on human development in the town of Jambeiro/SP during the 2000s. The goal is to investigate whether the improvement in the local economy is allied to improvement in quality of life. The study is grounded in contemporary theories that conceptualize the terms growth and development. The research was based on the profile of economic growth through the change in GDP and GDP per capita and human development profile by change in the Human Development Index HDI of the city between the years 2000 and 2010. After the comparison between economic and social indicators, the results reveal that the economy grows significantly. However, in improvement in the quality of life of the population, it is observed advancement in education levels, but the social aspects are still composed of social exclusion, reflected in the unequal distribution of income among the population.
\end{abstract}

Keywords: Economic Growth. Human Development. Jambeiro.

Recebido: $20 / 07 / 2016$

Aprovado: 30/12/2016 


\section{INTRODUÇÃO}

O crescimento econômico regional pode ser observado através do cálculo do produto que a economia de uma região foi capaz de desenvolver em determinado período. Consequentemente, tal análise baseia-se fundamentalmente em variáveis quantitativas. Contudo, a perspectiva do crescimento econômico não aborda variáveis qualitativas do desempenho econômico em favor do desenvolvimento econômico sustentável, reproduzido em desenvolvimento humano ou social.

No decorrer das últimas décadas, é possível observar que o ideal de vida social e cultural, linear às dimensões econômicas e materiais, tem sido rejeitado. Neste cenário, ganha espaço o contexto de desenvolvimento social baseado em paradigmas tradicionais de crescimento e, também, da análise da perspectiva de como tal crescimento influencia a vida das pessoas. Sendo assim, é válido falar em crescimento econômico desde que seja discutido de que maneira a sociedade participa e se beneficia dele.

Neste sentido, consideramos que as alterações na composição do produto, da renda e da alocação de recursos advindos dos diversos setores econômicos devem estar organizadas de forma a incrementar os indicadores de bem estar econômico e social e dirigir a promoção do desenvolvimento humano.

Para Oliveira (2002), não faz sentido crescer economicamente sem que haja, em paralelo, o desenvolvimento social, que pode ser entendido como a realização pessoal dos indivíduos de um país ou região. Nesta linha, para alcançar um desenvolvimento pleno e sustentável, faz-se necessária a redução da exclusão social, caracterizada por desigualdade e pobreza.

Sabendo que a economia do município de Jambeiro/SP apresentou transformações significativas ao longo das últimas décadas, cabe averiguar os aspectos relacionados a esse processo de crescimento econômico e, em paralelo, analisar se o incremento na economia deste espaço geográfico está acompanhado pelo desenvolvimento humano.

Para tanto, a partir desta introdução, o trabalho está dividido em quatro seções. Na primeira seção, torna-se fundamental confrontar, através de uma revisão teórica, os conceitos de crescimento e desenvolvimento econômico, desenvolvimento humano e a forma de mensurá-los ao longo do tempo. Na segunda seção, estão expostos os procedimentos metodológicos que conduzem a pesquisa. A terceira seção é destinada a análise dos indicadores socioeconômicos do município de Jambeiro/SP no período compreendido entre 2000 e 2010. Por fim, na quarta seção é estabelecida a conclusão da pesquisa. 


\section{CONCEITO E MENSURAÇÃO DE CRESCIMENTO E DE DESENVOLVIMENTO}

Discussões acerca dos conceitos entre crescimento e desenvolvimento são bastante difundidas no meio acadêmico. Para alguns, o crescimento é considerado um sinônimo do desenvolvimento. Para outros, o crescimento é a condição indispensável para o alcance do desenvolvimento, mas não condição suficiente.

A esse respeito, Vieira (2009) afirma que os termos desenvolvimento e crescimento econômico são frequentemente utilizados em estudos acadêmicos de variadas áreas científicas como: economia, história, geografia, entre outras. Além disso, são comuns na mídia e na política. Entretanto, seja em qual for o ambiente, percebe-se a existência de uma confusão entre o uso dos termos desenvolvimento econômico, crescimento econômico e desenvolvimento social.

Para Sandroni (1994), crescimento econômico é caracterizado como o aumento da capacidade produtiva da economia, ou seja, da produção de bens e serviços de um país ou de uma área econômica. Para tanto, é calculado através da evolução anual do Produto Nacional Bruto - PNB ou pelo Produto Interno Bruto - PIB. Além disso, também pode ser indicado pelo crescimento da força de trabalho, pela receita nacional poupada e investida e pelo grau de aperfeiçoamento tecnológico de uma economia.

Na mesma linha, Pinho e Vasconcellos (2003) apontam o crescimento econômico como a elevação contínua do produto nacional, em sentido global ou per capita, ao longo de um período, isto é, eficiência do sistema produtivo de uma economia. Segundo Vasconcellos e Garcia (2004), quando se pretende avaliar o crescimento econômico, um dos indicadores utilizados é o PIB, que corresponde ao somatório de todos os bens e serviços finais produzidos dentro do território nacional em determinado período de tempo.

Quanto ao desenvolvimento econômico, de acordo com Sandroni (1994), caracterizase como o crescimento econômico acompanhado pela melhoria do padrão de vida da população e por alterações essenciais na estrutura econômica e social. Na mesma perspectiva, Oliveira (2002) afirma:

Desenvolvimento nada mais é que o crescimento - incrementos positivos no produto e na renda - transformado para satisfazer as mais diversificadas necessidades do ser humano, tais como: saúde, educação, habitação, transporte, alimentação, lazer, dentre outras (Oliveira, 2002, p. 40).

Para Souza (1993), o desenvolvimento econômico exige um ritmo de crescimento econômico contínuo e superior ao crescimento populacional, englobando mudanças 3 Revista Competitividade e Sustentabilidade - ComSus, Paraná, v. 3, n. 2, p. 1-13, Jul /Dez. 2016. 
estruturais e melhoria nos indicadores de qualidade de vida. Dessa forma, o crescimento apresenta-se como fator chave para solução dos problemas humanos e para o desenvolvimento. Entretanto, em definição mais detalhada, a questão é saber como as variações do crescimento econômico são distribuídas entre a população e se são frutos de investimentos em educação, habitação e outros fatores que contribuem para a melhoria de vida da população.

O que percebemos é que o foco simplesmente em indicadores econômicos não são capazes de refletir, totalmente, condições de melhora ou piora na qualidade de vida de uma população. Sendo assim, observa-se que é cada vez mais frequente a discussão sobre como as pessoas tem sido afetadas pelos processos de crescimento econômico, isto é, se os aumentos no produto e na renda total resultam na promoção do desenvolvimento humano. Neste aspecto, Haq (1995) afirma que acrescentar a dimensão humana ao desenvolvimento representa um conceito totalmente novo.

Partindo desta premissa, além da contextualização de crescimento e desenvolvimento econômico, cabe também a caracterização do desenvolvimento humano. Sobre este tema, o Programa de Desenvolvimento das Nações Unidas - PNUD expõe em seu Relatório de Desenvolvimento Humano de 1990:

Human development is a process of enlarging people's choices. In principle, these choice [sic] can be infinite and change over time. But at all levels of development, the three essential ones are for people to lead a long and healthy life, to acquire knowledge and to have access to resources needed for a decent standard of living. If these essential choices are not available, many other opportunities remain inaccessible (UNDP, 1990, p. 10).

Feijó et al. (2001) aponta que desde os anos 90, autoridades reconhecem ser fundamental a produção de informações que possibilitem a mensuração do desempenho de países e regiões com relação aos temas econômicos, sociais e ambientais ao longo do tempo. Neste sentido, o PNUD propõe, em 1990, o Índice de Desenvolvimento Humano - IDH para captar estatísticas sobre o desenvolvimento humano.

O cálculo do IDH parte do pressuposto de que, apesar do desenvolvimento humano ser um processo de ampliação das oportunidades do ser humano, três condições fundamentais fazem parte de todos os níveis de desenvolvimento. São elas: desfrutar de uma vida longa e saudável, adquirir conhecimento e ter acesso a recursos necessário para um padrão de vida decente (PNUD, 1996). 
De acordo com o UNPD (1990), IDH é composto por três elementos essenciais, a contar: longevidade (expectativa de vida ao nascer), conhecimento (taxa de alfabetização e média dos anos de escolaridade) e padrão de vida (PIB per capita ajustado pela Paridade do Poder de Compra).

Segundo Kroetz et al. (2010), para mensurar o grau de desenvolvimento humano de um município, utiliza-se o Índice de Desenvolvimento Humano Municipal - IDHM, composto pelos mesmos três elementos calculados pelo IDH. A avaliação destes indicadores consideram a privação das regiões em relação a cada uma das três variáveis estudadas, que apresentam escala de limite entre zero e um. Dessa forma, após seu cálculo, o desenvolvimento da região pode ser classificado em:

- Menor que 0,500 - baixo desenvolvimento humano

- Entre 0,500 e 0,800 - médio desenvolvimento humano

- Acima de 0,800 - alto desenvolvimento humano

Ainda sobre este tema, o UNPD (1990) salienta que a ligação entre o crescimento econômico e o progresso humano não é automático. Para tanto, o crescimento do PIB acompanhado por uma distribuição equitativa de renda é fundamentalmente o caminho mais efetivo para sustentar o desenvolvimento humano.

Sendo assim, citamos mais um indicador de mensuração de desenvolvimento humano com foco na distribuição de renda, o índice de Gini. De acordo com Kroetz et al. (2010), um dos pontos que tal coeficiente permite verificar é o grau de concentração de renda em uma economia. O índice varia de zero a um e, quanto mais próximo de um, maior a concentração na distribuição do produto. $\mathrm{O}$ uso deste indicador permite observar distorções na distribuição de renda nacional.

Percebe-se que, da mesma forma que observamos significados diferentes entre os termos crescimento e desenvolvimento, observamos que a mensuração destes aspectos também se diferem entre si, de modo que a avaliação do crescimento baseia-se em análises quantitativas e, em contrapartida, o desenvolvimento exige estudos voltados a uma análise de dados qualitativa. Portanto, para mensurar a linearidade entre crescimento econômico e desenvolvimento humano de determinado espaço territorial, deve-se realizar uma análise baseada em índices qualitativos e quantitativos.

5 Revista Competitividade e Sustentabilidade - ComSus, Paraná, v. 3, n. 2, p. 1-13, Jul /Dez. 2016. 


\section{PROCEDIMENTOS METODOLÓGICOS}

A presente pesquisa assume caráter quantitativo, considerando seu objetivo de identificar a existência ou não de relação entre variáveis privilegiadas. Em paralelo, sua metodologia é descritiva, de forma que investiga eventos, atitudes ou situações manifestas em determinada população (LIMA, 2008).

A técnica de coleta de dados está fundamentada no levantamento bibliográfico, que reúne toda bibliografia já tornada pública em relação ao tema de estudo. Para tanto, não consideramos esta técnica como repetição das fontes reunidas, mas sim, uma apuração do assunto, tratado sob um novo enfoque ou abordagem, de forma a atingir conclusões inovadoras (Lakatos; Marconi, 2003).

O foco da pesquisa é o estudo de indicadores econômicos e sociais do município de Jambeiro. Os indicadores econômicos, coletados no site da Fundação Sistema Estadual de Análise de Dados Estatísticos - SEADE, concentram-se no estudo dos indicadores de produto e renda, com o objetivo de traçar o perfil do crescimento econômico do município. Dessa forma, a análise está baseada nas variações do PIB e PIB per capita e na participação dos setores econômicos no total do valor adicionado ao longo da década de 2000.

Por outro lado, os indicadores sociais foram coletados a partir do Atlas do Desenvolvimento Humano no Brasil 2013. O estudo leva em consideração o Índice de Desenvolvimento Humano Municipal, onde o objetivo é traçar o perfil do desenvolvimento humano do município através da análise da evolução na qualidade de educação, saúde e distribuição de renda. Para tanto, assim como na análise de indicadores econômicos, analisamos a variação dos indicadores sociais ao longo dos anos de 2000 e 2010.

Por fim, a correlação dos indicadores econômicos e sociais, dentro da esfera de informações obtidas, objetiva concluir a investigação, de modo que seja possível avaliar se, dentro do espaço geográfico delimitado, o crescimento econômico e o desenvolvimento humano crescem na mesma escala.

\section{ASPECTOS DO CRESCIMENTO ECONÔMICO NO MUNICÍPIO DE JAMBEIRO}

O município de Jambeiro está localizado no estado de São Paulo e, de acordo com Vieira e Santos (2013), faz parte da microrregião Paraibuna/Paraitinga, que é formada pelos municípios de Cunha, Jambeiro, Lagoinha, Natividade da Serra, Paraibuna, Redenção da Serra e São Luiz do Paraitinga. Este conjunto de municípios tem como base econômica a 


\section{ASPECTOS DO CRESCIMENTO ECONÔMICO E DO DESENVOLVIMENTO HUMANO NO MUNICÍPIO DE JAMBEIRO ...}

agropecuária de subsistência e poucas atividades industriais e de serviços, exceto Jambeiro, onde na década de 90 instalaram-se várias indústrias ao longo da Rodovia dos Tamoios.

Para analisar o crescimento econômico do município de Jambeiro, observamos, primeiramente, na Tabela 1, a evolução do seu PIB ao longo da década de 2000 e, para fins de comparação, a média do PIB dos municípios que compõe a microrregião Paraibuna/Paraitinga no mesmo período.

Tabela 1 - PIB (em milhões de reais) dos municípios da microrregião Paraibuna/Paraitinga

\begin{tabular}{llll}
\hline Localidade & $\mathbf{2 0 0 0}$ & $\mathbf{2 0 1 0}$ & $\begin{array}{l}\text { Variação em } \\
\text { \% } \\
\mathbf{( 2 0 0 0 - 2 0 1 0 )}\end{array}$ \\
\hline Jambeiro & $\mathbf{1 8 4 , 2 1}$ & $\mathbf{7 0 6 , 8 1}$ & $\mathbf{2 8 3 , 7 0 \%}$ \\
\hline Cunha & 47,28 & 140,54 & $197,25 \%$ \\
\hline Paraibuna & 65,50 & 221,98 & $238,90 \%$ \\
\hline Lagoinha & 15,82 & 51,52 & $225,66 \%$ \\
\hline Natividade da Serra & 19,30 & 52,20 & $170,46 \%$ \\
\hline Redenção da Serra & 12,69 & 41,68 & $228,44 \%$ \\
\hline São Luiz do Paraitinga & 33,14 & 89,56 & $170,24 \%$ \\
\hline
\end{tabular}

Fonte: Elaboração pelos autores a partir dos dados do SEADE

Observa-se que, entre os municípios da microrregião Paraibuna/Paraitinga, Jambeiro é o que apresenta os maiores valores de PIB, bem como o maior percentual de variação de crescimento do PIB durante a década de 2000.

Paraibuna é o município que apresenta os valores mais próximos aos de Jambeiro em relação ao PIB. Porém, ainda assim, em 2010, o PIB do município de Jambeiro corresponde a 3,2 vezes o valor do PIB do município de Paraibuna. Em contrapartida, o município de Redenção da Serra, no mesmo período analisado, apresenta um PIB dezessete vezes menor do que o PIB do município de Jambeiro.

Destarte, a partir da análise da variação do PIB do município de Jambeiro e da comparação desta variação com os demais resultados encontrados nos municípios da microrregião em questão, percebe-se que é o município que mais cresce economicamente entre os demais municípios da microrregião Paraibuna/Paraitinga entre os anos 2000 e 2010.

De forma a entender como é composto o produto total do município de Jambeiro, observamos, no Gráfico 1, a participação dos diversos setores econômicos no total do valor adicionado ao PIB entre os anos de 2000 e 2010 no município de Jambeiro e na microrregião Paraibuna/Paraitinga.

7 Revista Competitividade e Sustentabilidade - ComSus, Paraná, v. 3, n. 2, p. 1-13, Jul /Dez. 2016. 
Gráfico 1 - Participação dos Setores Econômicos no Total do Valor Adicionado

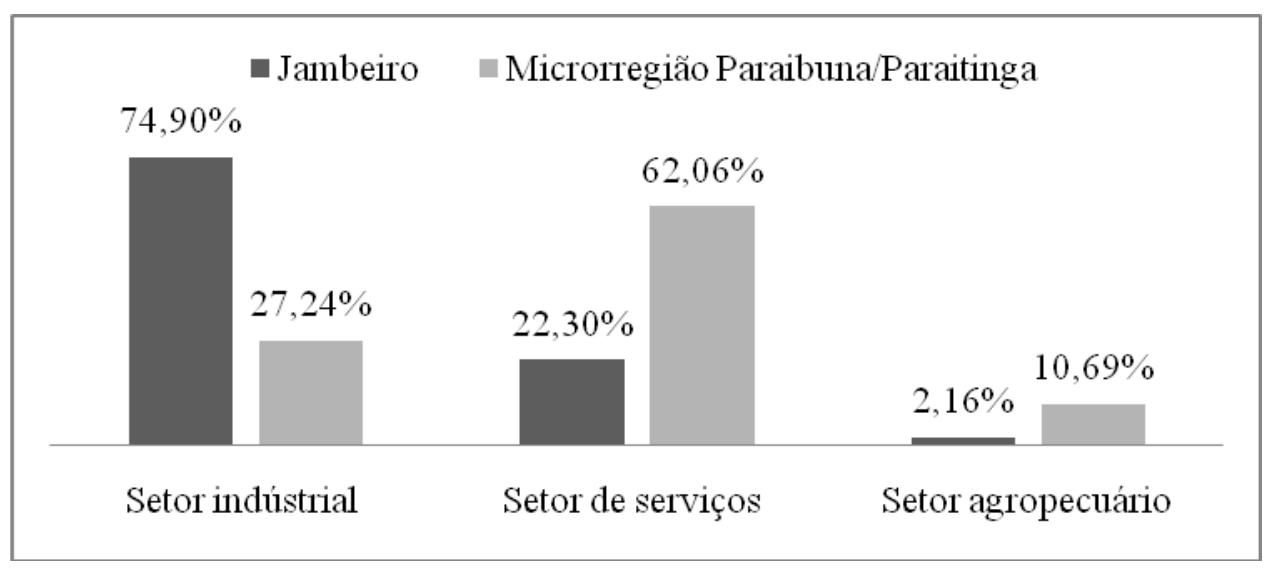

Fonte: Elaborado pelos autores a partir dos dados do SEADE

A partir da observação do gráfico, nota-se que, no município de Jambeiro, o crescimento econômico está refletido no setor industrial, isto é, a indústria representa o setor que mais agrega valor ao produto total, com um percentual de $74,90 \%$, contra $62,06 \%$ do setor de serviços e $2,16 \%$ do setor agropecuário. Estes percentuais mostram que este município apresenta uma composição de produto bastante diferente da média apresentada pelo conjunto de municípios de sua microrregião, onde o setor de serviços compõe a maior parte do produto total, seguido pela indústria e por fim, pela agropecuária.

Ademais, para aprofundar análise crescimento econômico, sob da ótica da renda, observaremos no Gráfico 2, o PIB per capita entre os anos 2000 e 2010, do município de Jambeiro e dos demais municípios da microrregião Paraibuna/Paraitinga, como mecanismo de verificação da distribuição do produto agregado produzido pelo município entre sua população.

Gráfico 2 - PIB per capita dos municípios da microrregião Paraibuna/Paraitinga em 2000 e 2010

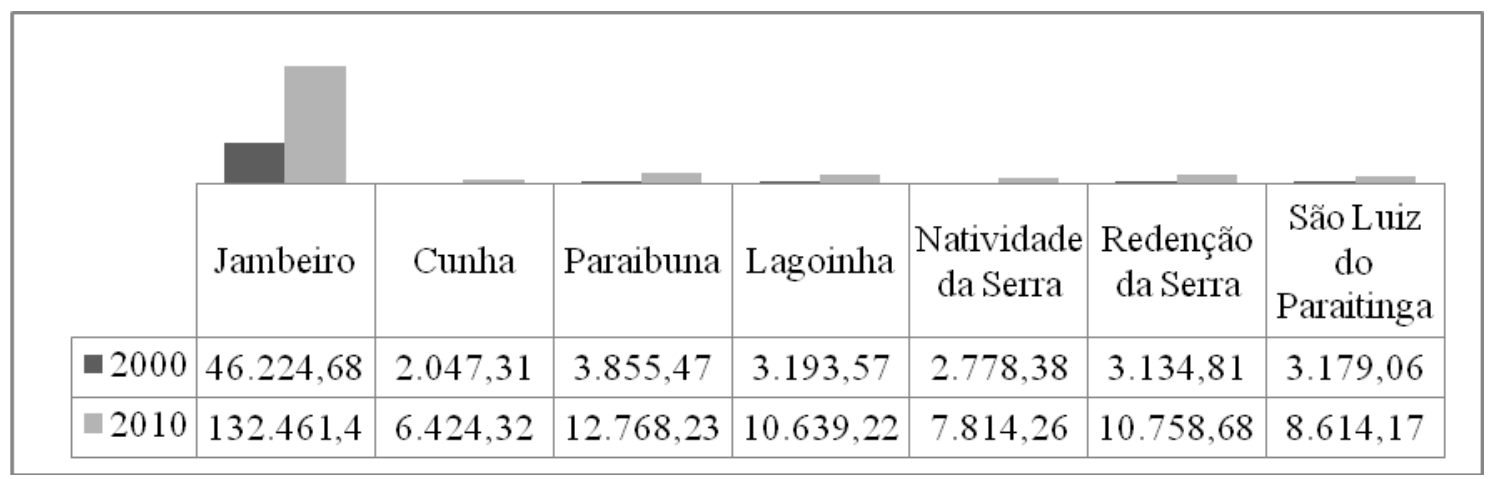

Fonte: Elaborado pelos autores a partir dos dados do SEADE

Observando o Gráfico 2, percebe-se que, além de apresentar o maior PIB entre os demais municípios da microrregião da qual faz parte, Jambeiro apresenta uma diferença 8 Revista Competitividade e Sustentabilidade - ComSus, Paraná, v. 3, n. 2, p. 1-13, Jul /Dez. 2016. 


\section{MUNICÍPIO DE JAMBEIRO ...}

exorbitante no que se refere ao valor de seu PIB per capita que, em 2010, atinge um valor de $\mathrm{R} \$ 132.461,44$. Isso significa que, em termos de distribuição do produto gerado ao longo dos anos estudados, Jambeiro praticamente triplicou sua renda por pessoa em um período de dez anos. Comparado aos demais municípios, Jambeiro possui um PIB per capita dez vezes maior que o de Paraibuna e vinte vezes maior do que Cunha, por exemplo.

\subsection{Aspectos do desenvolvimento humano no município de Jambeiro}

Para avaliar se o crescimento econômico reflete em melhoria da qualidade de vida para a população do município de Jambeiro, esta seção aborda a análise dos indicadores de desenvolvimento humano, onde analisamos o Índice de Desenvolvimento Humano Municipal - IDHM em sentido geral e em seu desdobramento nos subíndices de renda, educação e longevidade, agrupados na Tabela 2.

Tabela 2 - Evolução dos indicadores de desenvolvimento humano do município de Jambeiro

\begin{tabular}{lll}
\hline & $\mathbf{2 0 0 0}$ & $\mathbf{2 0 1 0}$ \\
\hline IDHM & $\mathbf{0 , 6 4 5}$ & $\mathbf{0 , 7 5 6}$ \\
\hline IDHM - Educação & $\mathbf{0 , 4 6 7}$ & $\mathbf{0 , 6 9 0}$ \\
\hline \% de 15 a 17 anos com Fundamental Completo & $56,53 \%$ & $67,19 \%$ \\
\hline \% de 18 a 20 anos com Médio Completo & $29,06 \%$ & $60,23 \%$ \\
\hline \% de 25 anos ou mais com Superior Completo & $5,59 \%$ & $8,18 \%$ \\
\hline IDHM - Longevidade & $\mathbf{0 , 8 5 2}$ & $\mathbf{0 , 8 6 0}$ \\
\hline Expectativa de vida ao nascer & 76,09 & 76,61 \\
\hline IDHM - Renda & $\mathbf{0 , 6 7 3}$ & $\mathbf{0 , 7 2 7}$ \\
\hline Percentual da renda apropriada pelos 20\% mais ricos & $58,17 \%$ & $55,44 \%$ \\
\hline Percentual de renda apropriada pelos $80 \%$ mais pobres & $41,83 \%$ & $44,56 \%$ \\
\hline Percentual de população extremamente pobre & $4,57 \%$ & $0,55 \%$ \\
\hline
\end{tabular}

Fonte: Elaborado pelos autores a partir do Atlas de Desenvolvimento Humano no Brasil 2013

A tabela aponta que, em geral, o Índice de Desenvolvimento Humano do município de Jambeiro passou de 0,645 em 2000 para 0,756 em 2010, números que apontam para um médio desenvolvimento humano ao longo da década de 2000. Especificamente, notamos que a variação no IDHM sofreu um aumento percentual de 17\% ao longo de uma década.

Quanto à educação, este foi o índice que mais cresceu entre 2000 e 2010, visto que encontramos um percentual de variação de $48 \%$ ao observar a alteração do resultado do índice de 0,467 em 2000 para 0,690 em 2010. Todavia, houve uma melhora pouco expressiva no percentual de jovens que concluíram o ensino fundamental, que variou $11 \%$. No entanto, 9 Revista Competitividade e Sustentabilidade - ComSus, Paraná, v. 3, n. 2, p. 1-13, Jul /Dez. 2016. 
encontra-se um resultado mais significativo no que diz respeito à conclusão do ensino médio, cujo percentual progrediu em 31\% entre 2000 e 2010. Quanto ao ensino mais avançado, em 2010 , o percentual de $8,18 \%$ aponta que ainda é reduzida a parcela da população, com 25 anos ou mais, que possui nível superior completo.

Com relação à saúde, o índice de longevidade indicou um valor de 0,852 em 2000, resultado alterado em apenas $0,9 \%$ ao longo de dez anos, quando atingiu, em 2010, um valor de 0,860 . Da mesma maneira, é bastante reduzida a alteração referente à expectativa de vida, que passou de 76,09 em 2000 para 76,61 em 2010.

Em se tratando da renda, nota-se desigualdade quanto sua distribuição entre a população. Percebe-se que, em 2000, 20\% da população mais rica da cidade concentra um percentual $58,17 \%$ de renda apropriada. No mesmo ano, $80 \%$ da população mais pobre apropria um percentual de $41,83 \%$ de renda. No ano de 2010 , há uma variação pouco significativa quanto a esta distribuição. Observa-se que, dez anos depois, os 20\% mais ricos apropriam $55,44 \%$ da renda, enquanto os $80 \%$ mais pobres atingem um percentual de $44,56 \%$ de apropriação de renda. Em contrapartida, ainda com relação à renda, observamos uma melhora no percentual de população extremamente pobre, que passa de 4,57\% em 2000 para $0,55 \%$ em 2010.

Para maior aprofundamento no estudo da distribuição da renda, avaliamos, no Gráfico 3, o coeficiente de Gini, que mede os aspectos da desigualdade social entre a população de Jambeiro e da microrregião de Paraibuna/Paraitinga entre 2000 e 2010.

Gráfico 3 - Índice de Gini

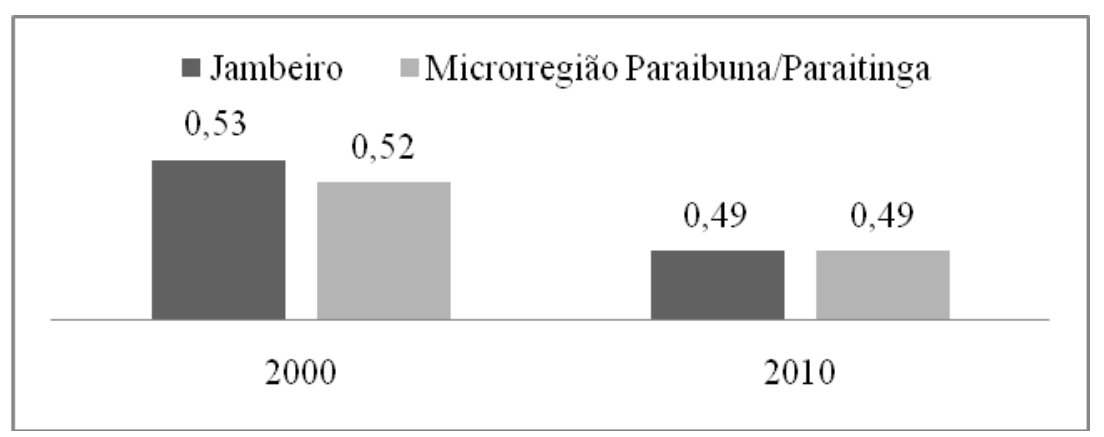

Fonte: Elaborado pelos autores a partir do Atlas de Desenvolvimento Humano no Brasil 2013

Constatamos que o crescimento do PIB per capita, analisado na seção anterior, está acompanhado de avanço pouco significativo no que tange a distribuição de renda no município de Jambeiro. Este fato pode ser observado quando se analisa que o índice de Gini passou de 0,53 em 2000 para 0,49 em 2010. Percebe-se que, com relação à distribuição de 


\section{MUNICÍPIO DE JAMBEIRO ...}

renda, o município de Jambeiro apresenta um resultado congênere ao resultado da microrregião Paraibuna/Paraitinga que, em 2010, também apresentou um índice de 0,49. Observa-se que neste aspecto social, diferentemente dos aspectos econômicos, Jambeiro não se destaca frente aos municípios de sua microrregião.

\section{CONCLUSÃO}

Percebe-se que, embora existam divergências entre os conceitos sobre crescimento e desenvolvimento, de fato, em certas perspectivas, eles se completam. Isso significa que alterações na composição do produto e alocação de diferentes recursos pelos diversos setores econômicos, devem estar ordenadas de forma a incrementar tanto indicadores de bem estar econômico quanto indicadores de bem estar social.

Destarte, para que o desenvolvimento humano seja alcançado, é necessária a redução da exclusão social, refletida na pobreza e desigualdade. De maneira prática, países ou regiões devem centrar esforços não apenas no crescimento do bolo, mas também em sua distribuição. Além disso, no que se refere ao desenvolvimento regional, devemos levar em conta que o progresso de uma região se dá através de sua capacidade em agrupar, ao mesmo tempo, maximização de recursos locais e melhoria da qualidade de vida de sua população.

Nesta linha, o município de Jambeiro/SP constituiu o espaço delimitado para uma análise que agrupou indicadores de crescimento econômico e de desenvolvimento humano. Para tanto, esta localidade foi apresentada, ao longo da década de 2000, como um espaço de intenso setor industrial que combinou, simultaneamente, variações expressivas em seu PIB e PIB per capita. Neste sentido, a eficiência na produção de riquezas, durante o período estudado, aponta crescimento econômico muito superior aos demais municípios que compõe a microrregião Paraibuna/Paraitinga.

Entretanto, o significativo avanço econômico está associado à melhoria da qualidade de vida da população de forma fracionada. Quando analisamos o IDHM de Jambeiro, percebemos que, entre 2000 e 2010, o município atingiu um nível de desenvolvimento mediano. Na educação, apesar de apresentar melhoras ao longo da década de 2000, ainda é possível observar deficiência nas taxas de conclusão do ensino fundamental, médio e superior. $\mathrm{Na}$ saúde, percebe-se um avanço pouco significativo nas variáveis de longevidade e expectativa de vida ao nascer. Em paralelo, ao analisar a renda, percebe-se que, tanto em 2000

11 Revista Competitividade e Sustentabilidade - ComSus, Paraná, v. 3, n. 2, p. 1-13, Jul /Dez. 2016. 
quanto em 2010, esta variável continua concentrada nas mãos da população $20 \%$ mais rica, isto é, a distribuição de renda entre a população é ineficiente.

Portanto, observa-se um descompasso entre o crescimento econômico e o desenvolvimento humano dentro do espaço estudado. De um lado, observa-se a economia crescendo a passos largos. Em contrapartida, no que se refere à melhoria da qualidade de vida da população, observa-se uma conjuntura social composta por exclusão social, refletida na má distribuição de renda entre a população.

\section{REFERÊNCIAS}

Feijó, C. A.; Young, C. E; Lima, F. C. G. C.; Galvão, O. J. A.; Ramos, R. L. O. Contabilidade social: o novo sistema de contas nacionais no Brasil. Rio de Janeiro: Campus, 2001.

Haq, M. U. Reflections on human development. New York: Oxford University Press. 1995.

Kroetz, M.; Ramos, I. S.; Cardoso, M. R.; Diel, A. S.; Schmitk, L.; Krause, R. Crescimento econômico x desenvolvimento: aspectos do crescimento econômico da região de Rio do Sul e o reflexo nas questões sociais. Revista Caminhos, Rio do Sul, v. 1, p. 25-45, out./dez. 2010.

Lakatos, E. M.; Marconi, M. A. Fundamentos de metodologia científica. 5. ed. São Paulo : Atlas. 2003.

Lima, M. C. Monografia: a engenharia da produção acadêmica. 2. ed. São Paulo : Saraiva. 2008.

Oliveira, G. B. Uma discussão sobre o conceito de desenvolvimento. Revista FAE, Curitiba, v.5, n.2, p.37-48, maio/ago. 2002.

Pinho, D. B.; Vasconcellos, M. A. S. Manual de economia. 3 ed. São Paulo: Saraiva. 1998.

Programa Das Nações Unidas Para O Desenvolvimento - PNUD. Relatório sobre o Desenvolvimento Humano no Brasil. Rio de Janeiro: IPEA/ PNUD. 1996.

Sandroni, P. Novo Dicionário de Economia. São Paulo: Editora Best Seller. 1994.

Souza, N. J. Desenvolvimento econômico. São Paulo: Atlas. 1993.

United Nations Development Programme - UNDP. Human Development Report 1990. New York: Oxford University Press. 1990.

Vasconcellos, M. A.; Garcia, M. E. Fundamentos de Economia. 2 ed. São Paulo: Saraiva. 2004.

Vieira, E. T. Industrialização e Políticas de Desenvolvimento Regional: o Vale do Paraíba Paulista na segunda metade do século XX. Tese de Doutorado em História Econômica. USP. 2009.

Vieira, E. T.; Santos, M. J. As ferramentas quantitativas e qualitativas na análise histórica do desenvolvimento regional: o Vale do Paraíba de 1970-2000. Revista Diálogos, Maringá, v. 17, n.1, p. 279-307, jan./abr. 2013. 


\section{NOTAS}

${ }^{1}$ Administradora e Mestre em Planejamento e Desenvolvimento Regional pela Universidade de Taubaté - E-mail: bruna_braun14@hotmail.com

2 Economista, Mestre Em Econômia e Doutor em Historia Econômica. Professor e Pesquisador da Univeridade de Taubaté - Programa de Mestrado em Planejamento e Desenvolvimento Regional e Professor do Centro Universidade Módulo. - E-mail: edson.trajano@pq.cnpq.br 